\title{
Quantification of transient increase of the blood-brain barrier permeability to macromolecules by optimized focused ultrasound combined with microbubbles
}

\author{
This article was published in the following Dove Press journal: \\ International Journal of Nanomedicine \\ 18 September 2014 \\ Number of times this article has been viewed
}

Lingyan Shi'

Paolo Palacio-Mancheno'

Joseph Badami²

Da Wi Shin'

Min Zeng'

Luis Cardoso'

Raymond $\mathrm{Tu}^{2}$

Bingmei $\mathrm{M} \mathrm{Fu}$

'Department of Biomedical Engineering, ${ }^{2}$ Department of Chemical Engineering, The City College of the City University of New York, New York, NY, USA
Correspondence: Bingmei M Fu Department of Biomedical Engineering, The City College of the City University of New York, 160 Convent Avenue, New York, NY I003I, USA

$\mathrm{Tel}+|2| 2650753 \mid$

$\mathrm{Fax}+\mathrm{I} 2126506727$

Email fu@ccny.cuny.edu

\begin{abstract}
Radioimmunotherapy using a radiolabeled monoclonal antibody that targets tumor cells has been shown to be efficient for the treatment of many malignant cancers, with reduced side effects. However, the blood-brain barrier (BBB) inhibits the transport of intravenous antibodies to tumors in the brain. Recent studies have demonstrated that focused ultrasound (FUS) combined with microbubbles (MBs) is a promising method to transiently disrupt the BBB for the drug delivery to the central nervous system. To find the optimal FUS and MBs that can induce reversible increase in the BBB permeability, we employed minimally invasive multiphoton microscopy to quantify the BBB permeability to dextran-155 kDa with similar molecular weight to an antibody by applying different doses of FUS in the presence of MBs with an optimal size and concentration. The cerebral microcirculation was observed through a section of frontoparietal bone thinned with a micro-grinder. About 5 minutes after applying the FUS on the thinned skull in the presence of MBs for 1 minute, TRITC (tetramethylrhodamine isothiocyanate)-dextran-155 kDa in $1 \%$ bovine serum albumin in mammalian Ringer's solution was injected into the cerebral circulation via the ipsilateral carotid artery by a syringe pump. Simultaneously, the temporal images were collected from the brain parenchyma 100-200 $\mu \mathrm{m}$ below the pia mater. Permeability was determined from the rate of tissue solute accumulation around individual microvessels. After several trials, we found the optimal dose of FUS. At the optimal dose, permeability increased by $\sim 14$-fold after 5 minutes post-FUS, and permeability returned to the control level after 25 minutes. FUS without MBs or MBs injected without FUS did not change the permeability. Our method provides an accurate in vivo assessment for the transient BBB permeability change under the treatment of FUS. The optimal FUS dose found for the reversible $\mathrm{BBB}$ permeability increase without $\mathrm{BBB}$ disruption is reliable and can be applied to future clinical trials.
\end{abstract}

Keywords: antibody delivery, multiphoton microscopy, in vivo cerebral microvessel permeability, rat brain

\section{Introduction}

Macromolecules such as monoclonal antibodies (mAbs) have attracted attention because of their potential clinical benefits. More recently, mAbs conjugated with radioisotopes, or radioimmunotherapy, administered into the cerebrospinal fluid (CSF), have proven safe in Phase I clinical trials for the treatment of leptomeningeal cancers (one type of brain cancer), and the patients survived for extended periods of time. ${ }^{1}$ While these studies have been shown to extend life, the methods are less than ideal because of their invasiveness and slow rate of diffusion from the CSF into the parenchyma. 
Access to the brain parenchyma is characteristically limited to cells lying close to the ventricles or infusion sites due to small diffusion distances of drugs and drug carriers through the interstitial space. ${ }^{2}$ The surface area of the blood-brain barrier (BBB) is 5,000 times that of the blood-CSF barrier, and therefore the $\mathrm{BBB}$ is considered to be the primary barrier controlling the uptake of drugs into the brain parenchyma. ${ }^{3}$ Thus, a more ideal route of administration would be through the systemic blood stream if obstacles related to BBB penetration of these macromolecules could be circumvented.

Among the numerous strategies to deliver therapeutic drugs into the central nervous system, the focused ultrasound (FUS) sonication combined with microbubbles (MBs) has been shown to be effective in transiently disrupting the BBB for noninvasive drug delivery. ${ }^{4-7}$ At appropriate acoustic power density (or pressure), burst repetition rate (BRR), duty cycle, sonication duration, and in the presence of MBs with proper materials, sizes, and concentrations, FUS can achieve noninvasive, selective, and localized disruption of the BBB without visible damage to the brain tissue..$^{8-11}$

FUS-induced BBB disruption has been widely assessed by using magnetic resonance imaging (MRI) or post-FUS histological examination. ${ }^{6,7,12-22}$ Indirect MRI collects the images of brain slices in the presence of an MRI contrast agent such as gadolinium to access the BBB disruption in different locations of the brain. Alternatively, post-FUS histological examination relates the $\mathrm{BBB}$ disruption to the amount of Evans blue extravasation in the brain tissue after the dye is intravenously injected into the blood circulation before or after sonication. However, the sub-millimeter spatial resolution of the MRI studies and the postmortem histological examinations only assess the relatively large leakage of the $\mathrm{BBB}$, and these methods are restricted to the exploration of a region that is much larger than a single microvessel with its surrounding tissue. Therefore, the BBB disruption assessed by the MRI and histological examination is more qualitative than quantitative. ${ }^{23,24}$

To overcome aforementioned limitations, two-photon microscopy can be employed to quantitatively access the enhanced permeability of the cerebral vasculature after FUS. ${ }^{25}$ In addition to micrometer spatial resolution, two-photon microscopy offers the advantage of deep tissue penetration, which is essential for the BBB permeability measurement. However, in the studies from Nhan et $\mathrm{al}^{25}$ the MBs for enhancing the FUS effect and the fluorescently labeled test solutes were injected via the animal tail vein. This administration route leads to the uneven distribution of MBs and uncertainty in the MB concentration in both the FUS-stimulated region and in the test solute concentration in cerebral microvessels, resulting in inaccurate assessments of the dynamics of FUSenhanced BBB permeability. In addition, their study only estimated the permeability index in a region of $\sim 500 \mu \mathrm{m} \times 500$ $\mu \mathrm{m}$ containing many types of blood vessels, not the true BBB permeability at the individual microvessel level.

The objective of the current study was to use our newly developed multiphoton microscopy ${ }^{23}$ to more accurately assess the FUS-induced BBB permeability change in vivo. To accomplish this aim, MBs were generated and selected for the optimal size in inducing reversible BBB disruptions. ${ }^{7,15,26,27}$ Subsequently, the MBs were injected via the carotid artery at a constant rate to achieve a constant concentration in the cerebral microvessels in the region of sonication, where the "optimal" concentration was based on previous studies. 6,7,21,28 After FUS in the presence of MBs, tetramethylrhodamine isothiocyanate (TRITC)-dextran-155 kDa, a model mAbs macromolecule, was injected via the carotid artery, and the BBB permeability was determined for an individual microvessel by a method described by Yuan et al. ${ }^{24}$ Our current study enabled us to find optimal FUS and MBs that can induce reversible increase in the BBB permeability to macromolecules. These findings will contribute to designing efficient and safe delivery strategies for the brain drug delivery, particularly for the delivery of antibodies.

\section{Materials and methods Microbubble preparation}

Lipid 1,2-distearoyl-sn-glycero-3-phosphocholine (DSPC; Avanti Polar Lipids Inc., Alabaster, AL, USA) was dissolved in chloroform (Thermo Fisher Scientific, Waltham, MA, USA). Stabilizing agent polyoxyethylene 40 stearate (PEG40S; Sigma-Aldrich, St Louis, MO, USA) was dissolved in filtered deionized water (18 M $\Omega$, Direct-Q; EMD Millipore, Billerica, MA, USA). All solutions were prepared using $1 \%$ bovine serum albumin (BSA) in Ringer's solution ( $\mathrm{pH} 7.4-7.45$ ) (see below for the composition), and glassware was previously cleaned with reagent alcohol (90:5:5 vol\% of ethanol/methanol/isopropanol; Sigma-Aldrich). ${ }^{29,30} \mathrm{MBs}$ were prepared as previously described. ${ }^{31}$

MBs having too large sizes may induce harmful effects such as blocking the blood flow in the pulmonary microvessels, while those having too small sizes may have no effects on enhancing the BBB permeability. ${ }^{10,11}$ In order to narrow down the size distribution of the MBs to near the optimal size of around $2 \mu \mathrm{m}$ in diameter, ${ }^{7,10}$ size isolation was performed by centrifugation. ${ }^{30}$ Briefly, MB solution was placed into a centrifuge (5702R; Eppendorf North America, 
Hauppauge, NY, USA) for 1 minute at $100 \mathrm{RCF}$ (relative centrifugal force) to remove bubbles larger than $\sim 4 \mu \mathrm{m}$. The infranatant was collected and centrifuged for another 1 minute at $200 \mathrm{RCF}$ to remove bubbles greater than $\sim 3 \mu \mathrm{m}$ in diameter. Then, the infranatant was collected again and centrifuged for 7 minutes at $300 \mathrm{RCF}$ to concentrate all bubbles with a diameter around $\sim 2 \mu \mathrm{m} .{ }^{26}$ The last step was repeated three times to ensure the complete removal of the remaining aggregates that did not form MBs. Size distributions were analyzed by using a laser particle size analyzer (LA-950; Horiba Inc., Irvine, CA, USA). Figure 1 shows the size distribution curve of the MBs generated by our technique, which have the optimal size of $\sim 2 \mu \mathrm{m}$ as an ultrasound contrast agent in in vivo ultrasound imaging and treatment applications..$^{7,10,11,26,32-34}$ The MB concentration prepared for injection was $\sim 1.7 \times 10^{6}$ bubbles $/ \mathrm{mL}$, which is in an optimal concentration range for reversible disruption of the $\mathrm{BBB}$ based on the study by Yang et al. ${ }^{7}$

\section{Animal preparation}

In vivo experiments were conducted on adult female Sprague Dawley rats (250-300 g, age 3-4 months; Hilltop Laboratory Animals Inc., Scottsdale, PA, USA). All procedures and the animal use were approved by the Institutional Animal Care and Use Committee of the City College of New York. Rats were anesthetized with pentobarbital sodium injected subcutaneously (initial dosage $65 \mathrm{mg} / \mathrm{kg}$ bodyweight). A heating pad was used to keep the rat at its body temperature. The depth of anesthesia was monitored for the absence of withdrawal reflex to toe pinch and absence of blink reflex. Anesthesia was further checked every 15 minutes during the experiment; an additional $3 \mathrm{mg} /$ dose pentobarbital was given when needed. At the end of the experiments, an overdose of pentobarbital $(>100 \mathrm{mg} / \mathrm{kg})$ was administered intravenously to euthanize the animal.

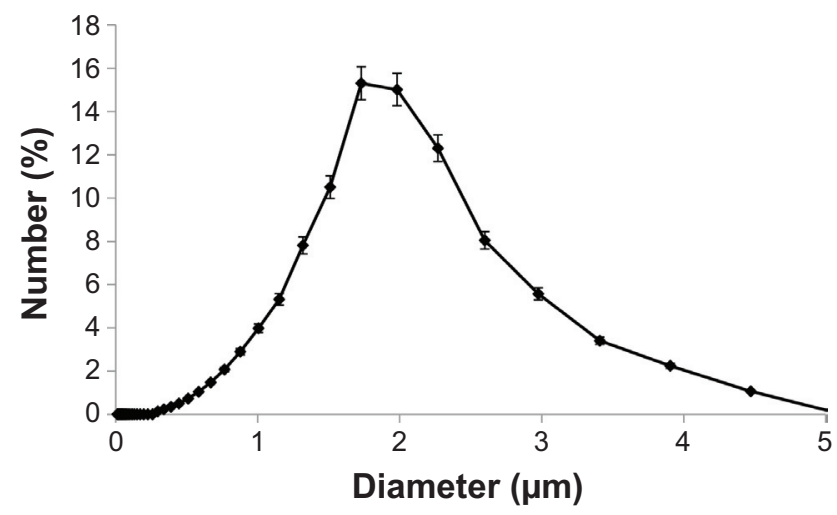

Figure I Size distribution of the microbubbles generated in the current study Notes: The error bars indicate the standard error of the mean.
The preparation of the rat skull observation area was the same as that previously described. ${ }^{23,24}$ Briefly, the skull in the region of interest (ROI) was exposed by shaving off the hair and cutting away the skin and connective tissue. A section of the frontoparietal bone (either left or right) was carefully ground with a high speed micro-grinder (0-50,000 rpm, DLT 50KBU; Brasseler USA, Savannah, GA, USA) until a part of it $(\sim 4 \mathrm{~mm} \times 6 \mathrm{~mm})$ became soft and translucent. During the process, artificial CSF (ACSF) at room temperature was applied to the surface of the skull to dispel the heat due to grinding. After grinding, the left or the right carotid artery was cannulated with PE50 tubing. The rat was then placed on a stereotaxic alignment system (SAS 597; David Kopf Instruments, Tujunga, CA, USA), and its head was fixed with two ear bars and a mouth clamp. After FUS treatment, the cerebral microvessels were observed under the objective lens of a multiphoton microscope through the thinned part of the skull and the BBB permeability was determined.

\section{Solutions and fluorescent test solute Mammalian Ringer's solution}

Mammalian Ringer's solution was used for all perfusates, which was composed of (in $\mathrm{mM}$ ) $\mathrm{NaCl} 132, \mathrm{KCl} 4.6, \mathrm{MgSO}_{4}$ 1.2, $\mathrm{CaCl}_{2} 2.0, \mathrm{NaHCO}_{3} 5.0$, glucose 5.5, and HEPES (2-[4(2-hydroxyethyl)piperazin-1-yl] ethanesulfonic acid) 20.

The $\mathrm{pH}$ was buffered to $7.40-7.45$ by adjusting the ratio of HEPES acid to base. In addition, both the washout solution and the fluorescent dye solution contained $10 \mathrm{mg} / \mathrm{mL} \mathrm{BSA}$ (A4378; Sigma-Aldrich). ${ }^{24}$ The solutions were made fresh on the day of use to avoid binding to the serum albumin.

\section{ACSF}

The ACSF solution composition was (in $\mathrm{mM}$ ) $\mathrm{NaCl} 110.5$, $\mathrm{KCl} 4.7, \mathrm{CaCl}_{2} 2.5, \mathrm{KH}_{2} \mathrm{PO}_{4} 1.1, \mathrm{MgSO}_{4} \cdot 7 \mathrm{H}_{2} \mathrm{O} 1.25, \mathrm{NaHCO}_{3}$ 25, and HEPES $15,{ }^{35}$ and the solution was buffered to $\mathrm{pH} 7.4 \pm 0.5$.

\section{Test solute}

TRITC-dextran-155 kDa (molecular weight 155,000, Stokes radius $\sim 8.5 \mathrm{~nm}$; Sigma-Aldrich) was used at the concentration of $1 \mathrm{mg} / \mathrm{mL}$ in $1 \%$ BSA in Ringer's solution. The concentration of $1 \mathrm{mg} / \mathrm{mL}$ was in the linear range $(0-2 \mathrm{mg} / \mathrm{mL})$ of the concentration versus intensity curve, which was calibrated in vitro using the same settings as in the in vivo experiments.

All the solutions were oxygenated by bubbling with the compressed gas composed of $95 \%$ oxygen and $5 \%$ carbon dioxide (Airgas; Bethlehem, PA, USA) for 5 minutes before being injected into the cerebral circulation. 


\section{Ultrasound equipment and calibration}

A spherical 1-MHz FUS transducer (Ws50-P50; Ultran, State College, PA, USA) with a focal length of $50 \mathrm{~mm}$ and a focal area diameter of $3 \mathrm{~mm}$ was used in this study. The power was generated by a function generator (AFG3251; Tektronix Inc., Beaverton, OR, USA). An ultrasound power meter (UPMDI-1AV; Ohmic Instruments, Easton, MD, USA) was used to measure the acoustic pressure generated by the ultrasound transducer in a degaussed water bath at room temperature. The acoustic pressure measured was automatically converted into power by the power meter system.

To determine the relationship of ultrasound power as a function of BRR (in kilohertz) and peak-to-peak voltage (in volts), powers were measured at varying BRRs $(200-1,000 \mathrm{kHz})$ and voltages $(1-5 \mathrm{~V})$. The duty cycle was kept constant at $5 \%$ for tested pulses from 200 to $1,000 \mathrm{kHz}$. Figure $2 \mathrm{dem}-$ onstrates the calibration results of the acoustic power density (power per unit focus area) versus voltage and BRR for the devices used in the current study. Dots are measured data, and the surface is the best fitting.

\section{FUS sonication with MBs}

Figure 3A illustrates the setup of ultrasound sonication. The ultrasound probe was placed in a custom-made clear acrylic adapter and was attached to a stereotaxic alignment system. The adapter allowed placement of the ultrasound probe in one end and a rubber-latex sheet (300-series, North; McMaster Carr, Robbinsville, NJ, USA) in the other. The reservoir water was degassed with a vacuum pressure pump (Air Cadet 4201901; Thermo Fisher Scientific) at room temperature $\left(25^{\circ} \mathrm{C}\right)$. By implementing the stereotaxic alignment system, the focal area of the ultrasound probe was easily and accurately located over the rat's head by softly pressuring the latex-sheet water reservoir over the thinned skull. Formation of air bubbles or air layers between the rat's skull and the rubber-latex sheet was avoided by applying an ultrasound transmission gel (National Medical Alliance, Carmel, IN, USA). After the rat was placed on the stereotaxic instrument and its head was fixed, the FUS sonication was applied to the brain through the thinned skull, while the MBs were injected simultaneously into the carotid artery at a constant flow rate $\sim 3 \mathrm{~mL} / \mathrm{min}$ by a syringe pump. We applied ultrasound at several acoustic powers (in a range of $\sim 2.85-15.00 \mathrm{~W} / \mathrm{cm}^{2}$ ) with different BRRs and voltages (Figure 2) to find the proper FUS dose for a reversible increase of the BBB permeability in the presence of our MBs.

\section{Two-photon microscopy and image collection}

The microvessels were observed with a $40 \times$ lens (water immersion, NA 0.8; Olympus Corporation, Tokyo, Japan), and the 12-bit images were collected by a two-photon microscopy system (Ultima; Prairie Technologies Inc., Middleton, WI, USA). For TRITC-dextran-155 kDa, the excitation wavelength of the two-photon microscope was set to $840 \mathrm{~nm}$.

After ultrasound sonication for $\sim 1$ minute in the presence of MBs, ${ }^{6,7}$ the rat was immediately placed under the objective of the two-photon microscope. The syringe was replaced by the one filled with TRITC-dextran- $155 \mathrm{kDa}$ solution (tracer) (Figure 3B). The tracer solution was then introduced into the cerebral circulation via the carotid artery at a constant flow rate of $\sim 3 \mathrm{~mL} / \mathrm{min}^{23,36}$ for 1 minute in an interval of 5 minutes. Simultaneously, the images of the ROI containing the microvessels and the surrounding brain tissue were captured for 1 minute at 5-minute intervals up to 25 minutes. The images of $239 \mu \mathrm{m} \times 239 \mu \mathrm{m}$

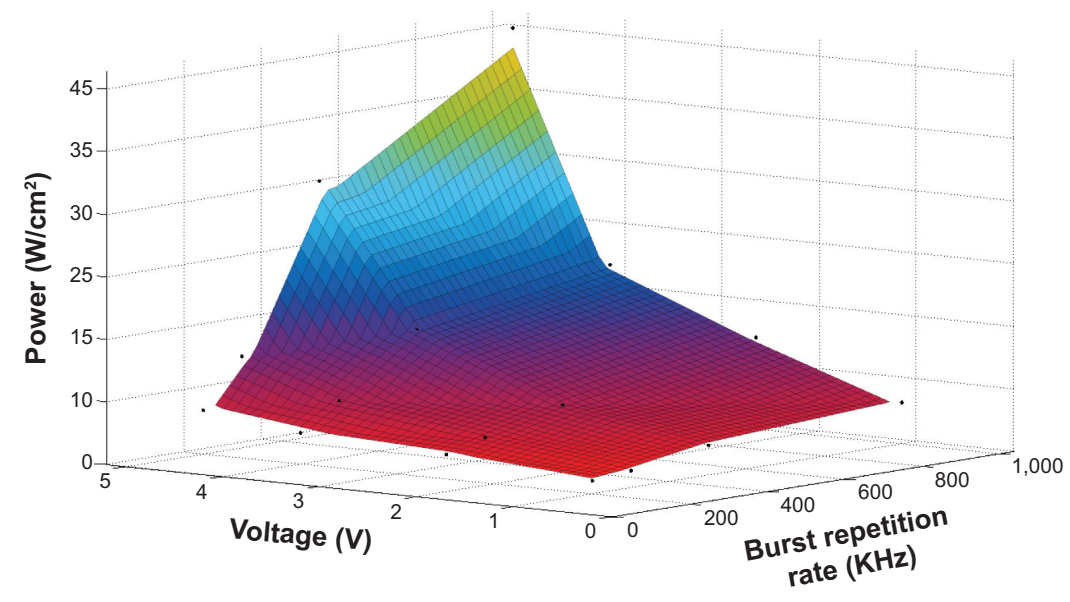

Figure 2 Calibration results for the acoustic power density as a function of voltage and burst repetition rate for the focused ultrasound transducer used in the current study. 
A

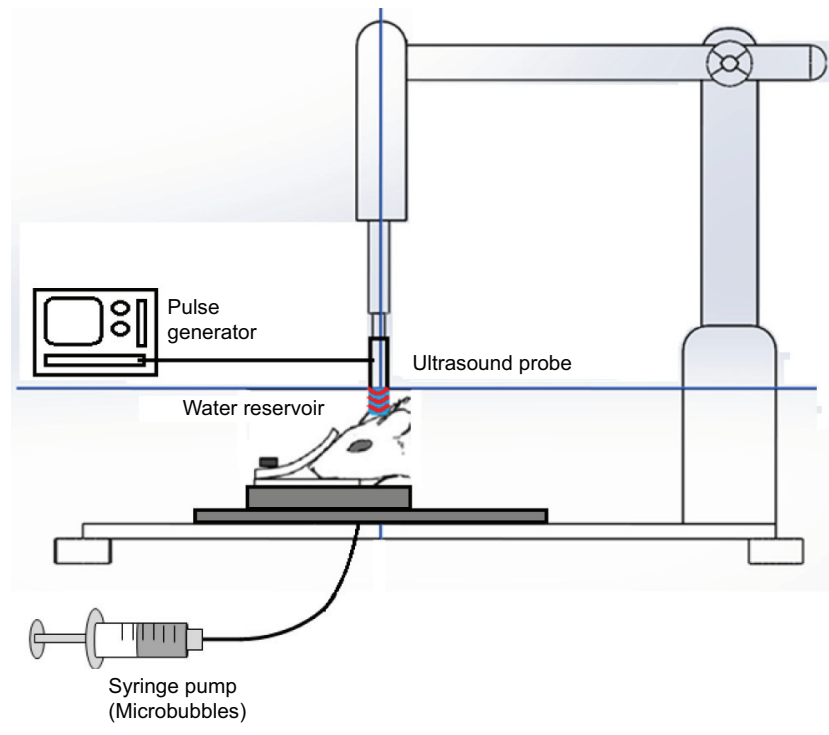

B

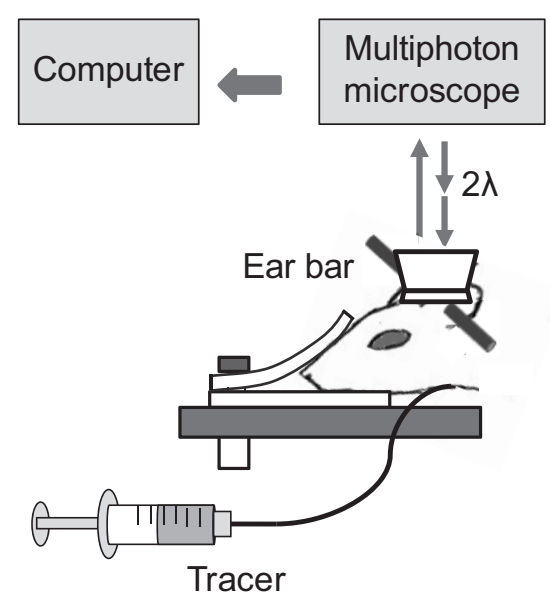

C

FUS and MB

injection

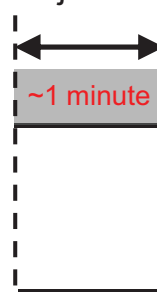

$\rightarrow$

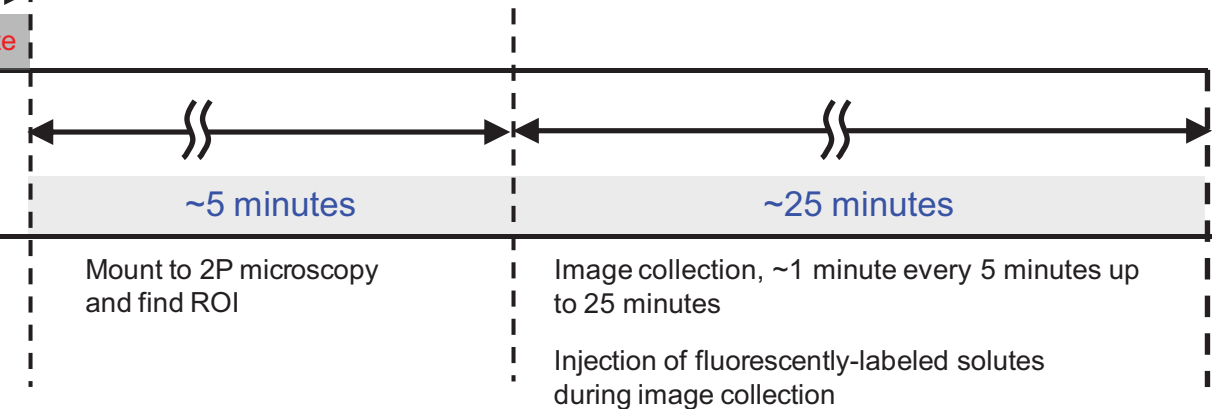

Figure 3 Illustrations of experimental setups.

Notes: (A) Schematic of the setup for the ultrasound sonication and MB injection to the cerebral circulation. Sonication on the thinned skull region starts simultaneously while the MBs are injected through the carotid artery at a rate of $\sim 3 \mathrm{~mL} / \mathrm{min}$. (B) Schematic of the blood-brain barrier solute permeability measurement by multiphoton microscopy. While TRITC-dextran- $155 \mathrm{kDa}$ (tracer) is injected through the carotid artery at a rate of $\sim 3 \mathrm{~mL} / \mathrm{min}$, the images of the ROI containing several microvessels and the surrounding brain tissue are collected. Permeability is determined off-line by analyzing the collected images. (C) Experimental timeline and protocol for the FUS sonication and blood-brain barrier permeability measurement.

Abbreviations: 2P, two-photon; FUS, focused ultrasound; ROI, region of interest; MB, microbubble; TRITC, tetramethylrhodamine isothiocyanate.

(512×512) were collected at a rate of $\sim 1$ second per image. The corresponding resolution is $0.467 \mu \mathrm{m} \times 0.467 \mu \mathrm{m}$. The collected images were then transferred to an image acquisition and analysis workstation to determine the BBB solute permeability.

\section{Experimental protocol}

Figure 3C summarizes the experimental protocol for FUS effects on the BBB solute permeability. The MBs at a concentration of $\sim 1.7 \times 10^{6}$ bubbles $/ \mathrm{mL}$ were injected via rat carotid artery at a constant speed of $\sim 3 \mathrm{~mL} / \mathrm{min}$, the normal blood flow velocity at the carotid artery; after 10-15 seconds, the MBs reach the microvascular bed $\sim 100-200 \mu \mathrm{m}$ below the pia mater, ${ }^{23}$ and the FUS sonication was administrated for 1 minute. After sonication, the rat head was mounted into the multiphoton microscope, and the ROI with cerebral microvessels was found. This took about 5 minutes. Then, the images for determining the BBB solute permeability were collected for 1 minute at a rate of one image per second in a 5 -minute interval, up to 25 minutes. For comparison, three control experiments were performed: 1) measurement of permeability in the presence of $1 \%$ BSA in Ringer's solution only, no MBs and FUS; 2) same protocol, with only MBs; 3) same protocol, with only FUS. 


\section{Determination of the BBB solute permeability}

We used the similar method as in our previous study for permeability of pial microvessels ${ }^{24}$ to determine permeability of cerebral microvessels $\sim 100-200 \mu \mathrm{m}$ below the pia mater. Most of the microvessels chosen were post-capillary venules of $15-40 \mu \mathrm{m}$ diameter for the permeability measurement, and around $1 / 4$ of the microvessels were capillaries. ${ }^{24,35}$ The reasons that we currently only measured the permeability of post-capillary venules and capillaries were: 1 ) to avoid the influence of smooth muscle cells at arteries, arterioles, and large venules, which would contract under stimuli and affect the permeability measurement; ${ }^{35} 2$ ) our previous studies on the BBB permeability were also conducted on this type of post-capillary venules. ${ }^{23,24}$

The permeability was determined off-line from the pre-collected images by using ImageJ (National Institutes of Health, Bethesda, MD, USA). Figure 4A shows a typical image of an ROI $(\sim 239 \mu \mathrm{m} \times 239 \mu \mathrm{m})$ with a couple of microvessels and surrounding brain tissue. The total fluorescence intensity in a rectangular window including a vessel lumen and the surrounding tissue (Figure 4A) was measured by ImageJ. The measuring window was $\sim 50-100 \mu \mathrm{m}$ long and $\sim 30-60 \mu \mathrm{m}$ wide and was set at least $10 \mu \mathrm{m}$ from the base of the bifurcation to avoid solute contamination from the side arms. The criteria for the size and placement of the measuring window were 1) the vessel segment is straight,
2) the dye does not spread out of the window during the time for permeability measurement (10-60 seconds), and 3) no dye contamination from the neighboring vessels into the window. When the criteria were satisfied, permeability was determined using the equation, ${ }^{24,37}$

$$
P=1 / \Delta I_{0} *(\mathrm{~d} I / \mathrm{d} t)_{0} * r / 2
$$

where $P$ is permeability, $\Delta I_{0}$ is the step increase of the fluorescence intensity in the window when the dye just fills up the vessel lumen (Figure 4B), $(\mathrm{d} I / \mathrm{d} t)_{0}$ is the slope of the increasing curve of the total intensity $I$ versus time $t$ when the solute further transfers into the surrounding tissue, and $r$ is the vessel radius.

\section{Corrections for influence of red blood cells, free dye, and solvent drag on BBB permeability}

The fluorescence dye solution was injected into the brain at the rate of $3 \mathrm{~mL} / \mathrm{min}$, the same as the normal blood perfusion rate. ${ }^{36,38}$ Although at this perfusion rate the blood was assumed to be replaced by the fluorescence solution, there was still residue blood (red blood cells [RBCs]) in the cerebral microvessels, which would overestimate the measured BBB permeability by $\sim 11 \%$, as estimated in Yuan et al. ${ }^{24}$

In addition to RBCs, free dye would overestimate the permeability to fluorescently labeled solutes..$^{24,39}$ The influence
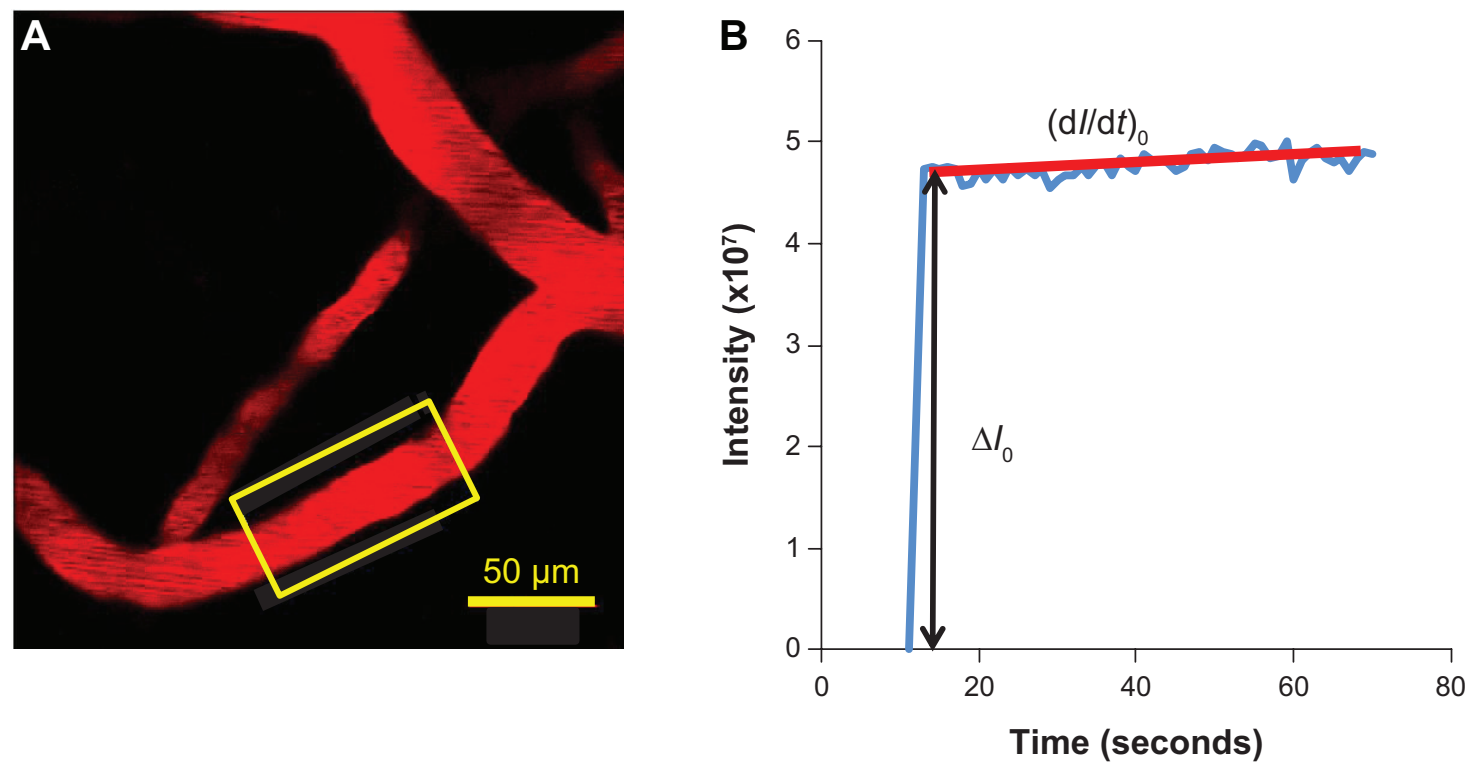

Figure 4 Determination of the blood-brain barrier solute permeability.

Notes: (A) Illustration of the scanning region comprising several microvessels $\sim 100-200 \mu \mathrm{m}$ below the pia mater. The region area is $\sim 239 \mu \mathrm{m} \times 239 \mu \mathrm{m}$. The yellow frame enclosed area is the region of interest used to determine the blood-brain barrier permeability to a solute. (B) Total fluorescence intensity in the region of interest as a function of perfusion time. Fluorescence intensity in the figure is proportional to the total mass of solute accumulated in the measuring region surrounding the microvessel. The slope of regression line over the initial linear accumulation $(\mathrm{d} l / \mathrm{d} t)_{0}\left(\right.$ red line) is used to determine permeability $P=I / \Delta I_{0} *(\mathrm{~d} / / \mathrm{d} t)_{0} * r / 2$, where $\Delta I_{0}(\mathrm{black}$ line with arrowheads) is the step intensity increase when the dye just fills up the vessel lumen, and $r$ is the radius of the vessel. 
of the free dye on the solute permeability was estimated by using equation, ${ }^{39}$

$$
P^{\text {correct }}=[1 /(1-\mathrm{F})] P^{\text {measure }}-[\mathrm{F} /(1-\mathrm{F})] P^{\text {freedye }},
$$

where $P^{\text {measure }}$ was measured permeability; $P^{\text {freedye }}$ was measured permeability of $\mathrm{NaF}\left(14.6 \times 10^{-7} \mathrm{~cm} / \mathrm{s}\right),{ }^{23}$ since the molecular weight of TRITC (479) is close to that of $\mathrm{NaF}$ (376); $\mathrm{F} \sim 0.3 \%$ was the intensity ratio of the free dye filtrate to the original fluorescently labeled solution for TRITC-dextran-155 kDa; and $P^{\text {correct }}$ was the corrected solute permeability.

The above apparent permeability $P$ corrected for the RBCs and free dye still overestimates the true diffusive solute permeability $P_{d}$ due to the coupling of solute flux with water flow (solvent drag). The $P_{d}$ for TRITC-dextran-155 kDa was calculated by using the following equations, ${ }^{39,40}$

$$
\begin{gathered}
P=P_{d} \frac{p_{e}}{\exp \left(p_{e}-1\right)}+L_{p}(1-\sigma) \Delta p_{e f f}, \\
P_{e}=\frac{L_{p}(1-\sigma) \Delta p_{e f f}}{P_{d}},
\end{gathered}
$$

where $P$ is the measured apparent permeability; $P_{e}$ is the Péclet number; $L_{p}$ is the hydraulic conductivity of the microvessel, which is $\sim 2.0 \times 10^{-9} \mathrm{~cm} / \mathrm{s} / \mathrm{cm} \mathrm{H}_{2} \mathrm{O}$ for the cerebral microvessels; ${ }^{23,41} \sigma$ is the reflection coefficient of the microvessel to the solute, and $\Delta p_{\text {eff }}$ is the effective filtration pressure across the microvessel wall, obtained from

$$
\Delta p_{\text {eff }}=\Delta p-\sigma^{\text {albumin }} \Delta \pi^{\text {albumin }}-\sigma^{\text {dye-solute }} \Delta \pi^{\text {dye-solute }},
$$

where $\Delta p$ and $\Delta \pi$ are the hydrostatic and oncotic pressure differences across the microvessel wall. The superscript dye-solute is TRITC-dextran- $155 \mathrm{kDa} ; \sigma$ of rat cerebral microvessels to the test solutes were estimated based on previous studies ${ }^{24}$ according to the molecule sizes; $\sigma^{\text {dextran }-155 \mathrm{kDa}}$ was estimated to be $0.96 ; \Delta p$ in the cerebral microvessel was $\sim 10 \mathrm{~cm} \mathrm{H}_{2} \mathrm{O}$; and $\Delta \pi^{\text {albumin }}$ was $3.6 \mathrm{~cm} \mathrm{H_{2 }} \mathrm{O}$ for $10 \mathrm{mg} / \mathrm{mL} \mathrm{BSA.}^{24}$

\section{Data analysis and statistics}

The BBB permeability to dextran-155 $\mathrm{kDa}$, measured at a specific time in the control group was used to normalize those in the test and sham control groups at the same time. Data were presented as mean \pm standard error unless otherwise specified. Significance was assumed for probability level $P<0.05$ using a two-way analysis of variance followed by a Tukey's post hoc analysis (JMP; SAS Institute Inc., Cary, $\mathrm{NC}, \mathrm{USA}$ ).

\section{Results}

The ultrasound power (voltage, BRR, and duty cycle), sonication duration, $\mathrm{MB}$ size, and $\mathrm{MB}$ concentration are important factors in controlling the BBB-disruption levels and determining whether the increased BBB permeability is reversible. ${ }^{6,7,11,15,21,25}$ Based on prior literature, ${ }^{6,7,10,21}$ we fixed the MB size to $\sim 2 \mu \mathrm{m}, \mathrm{MB}$ concentration to $\sim 1.7 \times 10^{6}$ bubbles $/ \mathrm{mL}$, and sonication time to $\sim 1$ minute. Subsequently, we adjusted the voltage and BRR while keeping the duty cycle constant at $5 \%$ to generate different acoustic powers. Figure $5 \mathrm{~A}$ shows a disrupted BBB after $\sim 5$ minutes by applying a power of $\sim 13.1 \mathrm{~W} / \mathrm{cm}^{2}$ (voltage $=4 \mathrm{~V}$ and $\mathrm{BRR}=500 \mathrm{kHz}$ ) for 1 minute. We then reduced the power to $\sim 6.3 \mathrm{~W} / \mathrm{cm}^{2}$ (voltage $=2.5 \mathrm{~V}$ and $\mathrm{BRR}=500 \mathrm{kHz}$ ). Figure 5B shows the image of the cerebral microvessels taken after $\sim 20$ minutes by applying 1-minute sonication at this dose. Although there was no visible BBB disruption, the increased permeability was not reversible in $\sim 60$ minutes (the measurement was stopped after 60 minutes). The corresponding BBB permeability measured after 5, 10, 30 , and 60 minutes was found to be $27.1 \times 10^{-7}, 23.5 \times 10^{-7}$, $23.6 \times 10^{-7}$, and $19.6 \times 10^{-7} \mathrm{~cm} / \mathrm{s}$, respectively. These values are more than ten-fold greater than the control values without FUS treatment (Table 1). Reducing the power to $3.4 \mathrm{~W} / \mathrm{cm}^{2}$ by decreasing the voltage to $1.25 \mathrm{~V}$, there was no change in the BBB permeability for 60 minutes. However, keeping the same power of $\sim 6.3 \mathrm{~W} / \mathrm{cm}^{2}$, but reducing the voltage to $1.25 \mathrm{~V}$ and increasing the BRR to $1,000 \mathrm{kHz}$, we were able to achieve a reversible $\mathrm{BBB}$ permeability increase in $\sim 30$ minutes. Figure $5 \mathrm{C}$ shows the image of the cerebral microvessels after $\sim 20$ minutes by applying this reversible acoustic dose for 1 minute. For comparison, Figure 5D shows the image of microvessels under the control condition without FUS and MBs. The fluorescence intensity in the surrounding tissue of the microvessels was noticeably higher in Figures $5 \mathrm{~B}$ and $\mathrm{C}$ than that in Figure 5D, indicating more tracers passing across the microvessel due to the FUS-induced permeability increase in the presence of MBs.

Figure 6 compares the BBB permeability to dextran$155 \mathrm{kDa}$, under controls and under the treatment of the optimized FUS $\left(6.3 \mathrm{~W} / \mathrm{cm}^{2}\right.$ when voltage $=1.25 \mathrm{~V}$ and $\mathrm{BRR}=1,000 \mathrm{kHz}$ ) in the presence of MBs. The solid line with filled squares in Figure 6 demonstrates the response of permeability to FUS in the presence of MBs as a function of time. At 5 minutes post-FUS, the mean permeability increased 

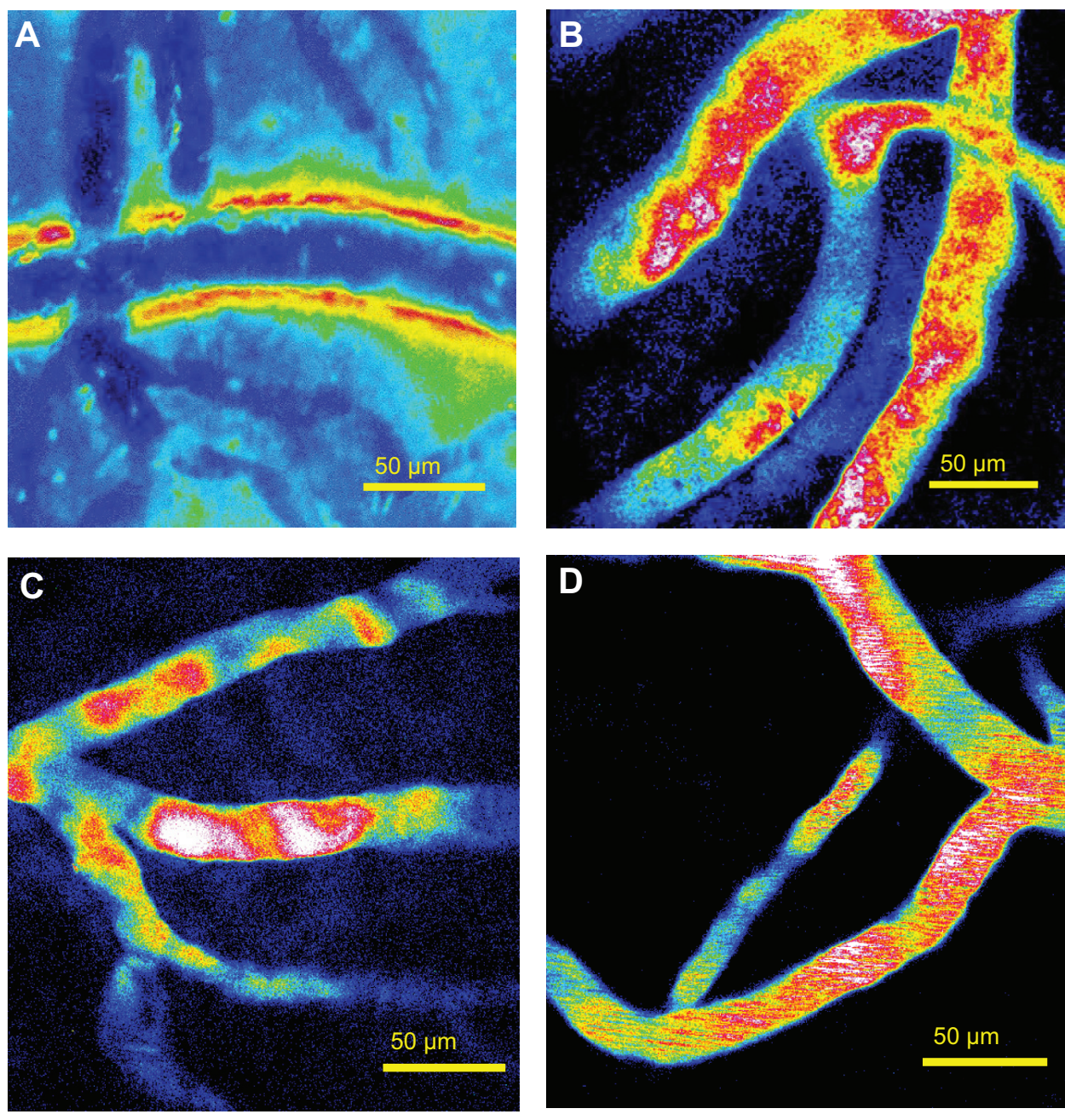

Figure 5 Representative images of the scanning region under various conditions.

Notes: (A) After $\sim 5$ minutes post-FUS at the dose of acoustic power density $13.1 \mathrm{~W} / \mathrm{cm}^{2}$ (voltage $=4 \mathrm{~V}, \mathrm{BRR}=500 \mathrm{kHz}$ ) with MB injection. (B) After $\sim 20 \mathrm{minutes}$ post-FUS at a lower dose of $6.3 \mathrm{~W} / \mathrm{cm}^{2}$ (voltage $=2.5 \mathrm{~V}, \mathrm{BRR}=500 \mathrm{kHz}$ ) with $\mathrm{MB}$ injection. (C) After $\sim 20$ minutes post-FUS at the same lower dose of $6.3 \mathrm{~W} / \mathrm{cm}^{2}$, but reduced voltage $=1.25 \mathrm{~V}$ and increased $B R R=I, 000 \mathrm{kHz}$, with $M B$ injection. (D) Under control condition, with neither ultrasound sonication nor MB injection.

Abbreviations: BRR, burst repetition rate; FUS, focused ultrasound; MB, microbubble.

Table I Cerebral microvessel permeability $(\mathrm{P})$ to TRITC-dextran-I55 kDa under various conditions at different time points and the corresponding corrected $\mathrm{P}$ for the RBCs, free dye, and solvent drag

\begin{tabular}{|c|c|c|c|c|c|c|c|}
\hline Group & $\begin{array}{l}\text { Vessel } \\
\text { radius }(\mu \mathrm{m}) \\
(\text { mean } \pm \mathrm{SE})\end{array}$ & $\begin{array}{l}\text { Time } \\
\text { (minutes) }\end{array}$ & $\begin{array}{l}P \text { (measured) } \\
\left(\times 10^{-7} \mathrm{~cm} / \mathrm{s}\right) \\
(\text { mean } \pm \mathrm{SE})\end{array}$ & $\begin{array}{l}P \text { (corrected } \\
\text { for } R B C) \\
\left(\times 10^{-7} \mathrm{~cm} / \mathrm{s}\right)\end{array}$ & $\begin{array}{l}P \text { (corrected } \\
\text { for } R B C \text { and } \\
\text { free dye) } \\
\left(\times 10^{-7} \mathrm{~cm} / \mathrm{s}\right)\end{array}$ & $\begin{array}{l}P_{d} \text { (corrected for } \\
\text { RBC, free dye, } \\
\text { and solvent drag) } \\
\left(\times 10^{-7} \mathrm{~cm} / \mathrm{s}\right)\end{array}$ & $\begin{array}{l}P_{d} / P \text { (corrected } \\
\text { for RBC and } \\
\text { free dye) }\end{array}$ \\
\hline \multirow{5}{*}{$\begin{array}{l}\text { Ultrasound } \\
\text { and bubble } \\
(n=15)\end{array}$} & \multirow[t]{5}{*}{$10.7 \pm 1.0$} & 5 & $19.57 \pm 4.49$ & 17.42 & 17.42 & 16.99 & 0.9752 \\
\hline & & 10 & $14.46 \pm 2.07$ & 12.87 & 12.87 & 12.86 & 0.9998 \\
\hline & & 15 & $11.02 \pm 2.92$ & 9.81 & 9.80 & 9.80 & 0.9997 \\
\hline & & 20 & $5.12 \pm 1.30$ & 4.55 & 4.54 & 4.54 & 0.9994 \\
\hline & & 25 & $2.34 \pm 0.6 \mathrm{I}$ & 2.08 & 2.07 & 2.07 & 0.9988 \\
\hline \multirow{2}{*}{$\begin{array}{l}\text { Bubble only } \\
(n=6)\end{array}$} & \multirow[t]{2}{*}{$8.1 \pm 1.5$} & 5 & $1.40 \pm 0.36$ & 1.25 & 1.23 & 1.23 & 0.9979 \\
\hline & & 25 & $1.38 \pm 0.47$ & 1.23 & 1.21 & 1.21 & 0.9979 \\
\hline \multirow{2}{*}{$\begin{array}{l}\text { Ultrasound } \\
\text { only }(n=6)\end{array}$} & \multirow[t]{2}{*}{$7.9 \pm 1.4$} & 5 & $1.51 \pm 0.33$ & 1.34 & 1.33 & 1.33 & $0.998 I$ \\
\hline & & 25 & I. $24 \pm 0.24$ & 1.10 & 1.09 & 1.09 & 0.9976 \\
\hline \multirow{3}{*}{$\begin{array}{l}\text { Control } \\
(n=6)\end{array}$} & \multirow[t]{3}{*}{$6.8 \pm 1.3$} & 0 & $1.13 \pm 0.19$ & 1.00 & 0.99 & 0.99 & 0.9974 \\
\hline & & 5 & $1.37 \pm 0.21$ & 1.22 & 1.21 & 1.21 & 0.9979 \\
\hline & & 25 & $1.72 \pm 0.17$ & 1.53 & 1.52 & 1.51 & 0.9983 \\
\hline
\end{tabular}

Abbreviations: TRITC, tetramethylrhodamine isothiocyanate; RBC, red blood cell; SE, standard error of the mean; $P_{\mathrm{d}}$, diffusive permeability. 


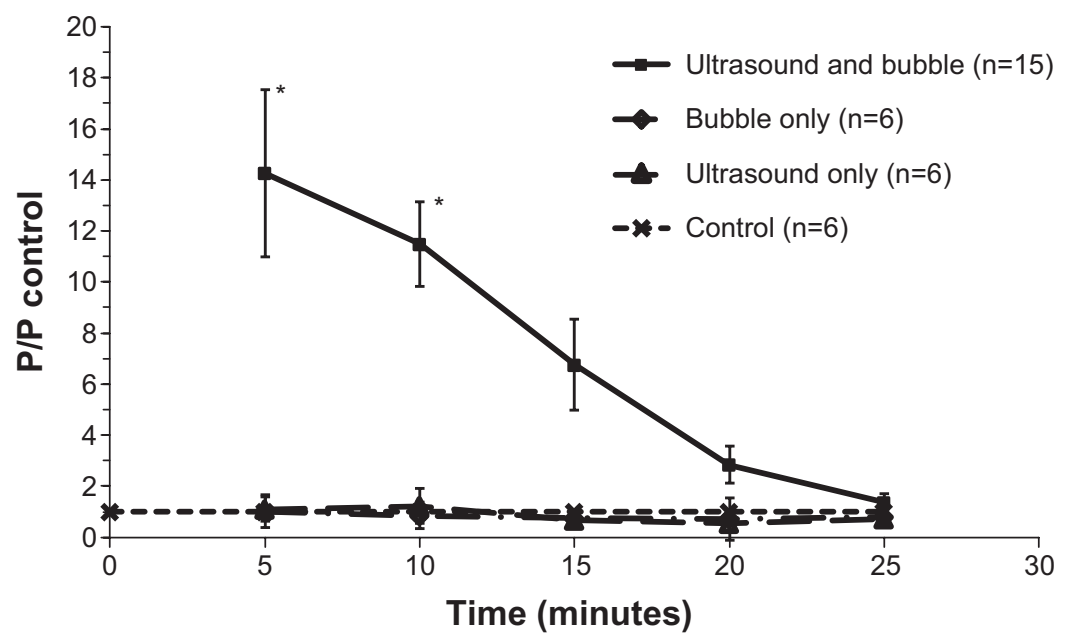

Figure 6 Normalized blood-brain barrier permeability as a function of time.

Notes: The solid line with $(\mathbf{\square})$ is for the treatment by ultrasound of $6.3 \mathrm{~W} / \mathrm{cm}^{2}$ (voltage $=1.25 \mathrm{~V}$, burst repetition rate $=1,000 \mathrm{kHz}$ ) in the presence of microbubbles. The dash-dot-dash line with $(\triangleleft)$ is for microbubbles only, with microbubble injection but without ultrasound sonication. The long dashed line with $(\Delta)$ is for ultrasound only, with ultrasound sonication in the absence of microbubbles. The short dashed line with $(\times)$ is for the control, with neither ultrasound nor microbubbles. $* P$-value $<0.05$ compared with the control.

Abbreviation: $\mathrm{P}$, permeability.

to $(19.6 \pm 4.5) \times 10^{-7} \mathrm{~cm} / \mathrm{s}(\mathrm{n}=15)$, a significant increase of $14.3( \pm 4.1)$-fold compared with the control $(P=0.025)$. The mean permeability at $10,15,20$, and 25 minutes was $(14.5 \pm 2.1) \times 10^{-7},(11.0 \pm 2.9) \times 10^{-7},(5.1 \pm 1.3) \times 10^{-7}$, and $(2.3 \pm 0.6) \times 10^{-7} \mathrm{~cm} / \mathrm{s}$, respectively, representing increases of $11.5( \pm 3.3, P=0.001), 6.8( \pm 2.1, P=0.052), 2.9( \pm 1.0, P=0.11)$, and $1.4( \pm 0.5, P=0.45)$-fold compared with the control group. At 15 minutes post-FUS, permeability started to recover values similar to the control; after 25 minutes, permeability completely returned to the value comparable to the control, indicating a reversible BBB permeability increase under this FUS dose in the presence of MBs.

For comparison, the permeability of the control group $(n=6)$ measured at $0,5,10,15,20$, and 25 minutes was $(1.1 \pm 0.2) \times 10^{-7},(1.4 \pm 0.2) \times 10^{-7},(1.4 \pm 0.3) \times 10^{-7}$, $(1.6 \pm 0.3) \times 10^{-7},(1.8 \pm 0.5) \times 10^{-7}$, and $(1.7 \pm 0.3) \times 10^{-7} \mathrm{~cm} / \mathrm{s}$, respectively. The permeability of that under only $\mathrm{MB}$ injection without sonication (bubble only, $\mathrm{n}=6$ ) at 5, 10, 15, 20, and 25 minutes was $(1.4 \pm 0.4) \times 10^{-7},(1.1 \pm 0.3) \times 10^{-7},(1.2 \pm 0.1) \times 10^{-7}$, $(1.3 \pm 0.9) \times 10^{-7}$, and $(1.4 \pm 0.5) \times 10^{-7} \mathrm{~cm} / \mathrm{s}$, respectively. The permeability of that under FUS in the absence of MBs ("ultrasound only", $\mathrm{n}=6$ ) at $5,10,15,20$, and 25 minutes was $(1.5 \pm 0.3) \times 10^{-7},(1.6 \pm 0.4) \times 10^{-7},(1.3 \pm 0.2) \times 10^{-7}$, $(1.1 \pm 0.1) \times 10^{-7}$, and $(1.2 \pm 0.2) \times 10^{-7} \mathrm{~cm} / \mathrm{s}$, respectively. They have no significant differences compared with the control group $(P>0.6)$.

Table 1 summarizes the measured permeability and its corrected value for the RBCs, free dye, and solvent drag under various conditions at specific times. Since the ratio of the diffusive permeability, $P_{d}$, to the apparent permeability corrected after RBC and free dye was more than $97.5 \%$, the solvent drag contribution to the BBB permeability to TRITC-dextran-155 kDa was negligible. In contrast, the solvent drag contribution to the macromolecule permeability is significant in rat mesenteric microvessels due to two orders of magnitude higher hydraulic conductivity. ${ }^{37}$ Table 1 also summarizes the mean radius of the microvessels for each group. Since there was no significant difference in the BBB permeability between the post-capillary venules and the capillaries $(P>0.09)$, we only gave a mean size for these two types of microvessels.

\section{Discussion}

Nhan et $\mathrm{al}^{25}$ used two-photon microscopy to investigate the reversible and localized $\mathrm{BBB}$ disruption induced by FUS in the presence of MBs by imaging the cerebral vasculature during $\mathrm{BBB}$ disruption and observing the extravasation of fluorescently labeled solutes in real-time in vivo. They found that the threshold acoustic pressure for a successful induction in BBB disruption was 0.4-0.6 MPa by combining the MBs with a similar size and a reduced concentration as those used in the current study. Under this dose of FUS and the MBs, they observed a visible $\mathrm{BBB}$ disruption in the cerebral microvasculature, which peaked around 5 minutes, especially for microvessels with diameter of 10-30 $\mu \mathrm{m}$. By using a formula in Dreher et $\mathrm{al}^{42}$ they converted the extravasation rate of the fluorescently labeled solutes to a global permeability index for the 
entire microvasculature, which peaked around 5 minutes and returned to the baseline in 20 minutes for test solutes, dextran-10k and -70k, in their study. Their estimated FUSinduced microvasculature permeability index changing patterns are similar to those in the individual microvessels under the current study. However, we found that under $0.43 \mathrm{MPa}\left(13.1 \mathrm{~W} / \mathrm{cm}^{2}\right)$ of our FUS, the wall of the individual microvessel was disrupted and could not go back to its baseline (Figure 5A). Even at a reduced power of $0.3 \mathrm{MPa}$ $\left(6.3 \mathrm{~W} / \mathrm{cm}^{2}\right)$ with a voltage of $2.5 \mathrm{~V}$, the permeability of the individual microvessel could not go back to its baseline value in $\sim 60$ minutes (Figure $5 \mathrm{~B}$ ).

The study from Nhan et $\mathrm{al}^{25}$ was invaluable in narrowing parameter space and defining a consistent methodology. However, the method in Nhan et $\mathrm{al}^{25}$ had some limitations. First, being injected via the tail vein, MBs would go back to the right ventricle of the heart, through the pulmonary circulation before they go into the systemic circulation and reach the cerebral microvessels. Along this journey, the MBs would be trapped in the pulmonary microvessels with smaller diameters, be diluted, and result in an uncertain concentration in brain microvessels. This would induce inaccurate assessment of proper parameters for the FUS which can induce transient BBB permeability increase. Second, the equation they used to determine the microvasculature permeability assumes a constant driving force that is represented by the fluorescent intensity difference between the intravascular and extravascular spaces along the microvessel. However, tail vein injection of the fluorescently labeled solutes might not achieve a constant driving force along the microvessel due to the plasma clearance. Third, because of the poor drainage in the brain tissue, the built-up extravascular tracers could not be cleared out in time and may induce a saturated extravascular concentration or an extravascular concentration which is higher than the intravascular one, resulting in an inaccurate assessment of microvascular permeability by using the formula in Dreher et al. ${ }^{42}$ Fourth, the region of $\sim 500 \mu \mathrm{m} \times 500 \mu \mathrm{m}$ from where they determined the permeability, contains many types of microvessels including arterioles and venules. Postcapillary venules and capillaries were suggested to be more suitable for permeability study since they are relatively less contractible or expandable under stimulations. ${ }^{35}$ These properties are necessary for accurate determination of the BBB permeability under FUS stimulations. ${ }^{35}$ Finally, their method can only give a relative permeability index, not the true solute permeability for a microvessel defined by the KedemKatchalsky equation. ${ }^{42}$ To overcome the aforementioned limitations, in our work we used multiphoton microscopy with a higher spatial resolution to accurately determine the BBB permeability for the individual post-capillary venule or capillary under the FUS treatment. By injecting both MBs and fluorescently labeled test solutes through the carotid artery, we can achieve a more constant concentration in the cerebral microvessels.

Previous studies have shown that the ultrasound power (voltage, BRR, and duty cycle), sonication duration, and MB size and concentration are important factors in controlling the BBB-disruption levels and determining whether or not the increased BBB permeability is reversible. ${ }^{6,711,15,21,25}$ Using MRI, the acoustic power threshold of opening the BBB was found to be $0.15-0.3 \mathrm{MPa} .{ }^{43}$ Whereas, the power threshold using two-photon microscopy was found to be $0.4-0.6 \mathrm{MPa} .{ }^{25}$ Using the same MB size and concentrations, we found that the FUS with the same power $\left(0.3 \mathrm{MPa}\right.$ or $\left.6.3 \mathrm{~W} / \mathrm{cm}^{2}\right)$ at the reduced voltage and increased $\mathrm{BRR}$ induced a reversible $\mathrm{BBB}$ permeability increase compared with that with higher voltage and lower BRR, which did not exhibit reversibility. The mechanism responsible for this is unknown. Under this range of the FUS power, the thermal effect is negligible. ${ }^{44}$ Other possible mechanisms for increasing the BBB permeability by FUS-driven MB activities in the cerebral microvessels include stable cavitation (stable small oscillation) and inertial cavitation (large amplitude oscillation). ${ }^{11,45,46}$ These activities can generate calcium transient, ${ }^{11,22,47}$ which may be responsible for the transient increase in the BBB permeability. ${ }^{48}$ The increased $\mathrm{Ca}^{2+}$ concentration in brain microvascular endothelial cells by FUS-driven $\mathrm{MBs}^{22}$ is similar to that induced by bradykinin, histamine, and adenosine triphosphate (ATP), which are known to transiently increase the BBB permeability. ${ }^{49,50}$ The increased magnitude and the temporal pattern in the permeability of an individual cerebral microvessel induced by the optimized FUS and MBs in the current study are similar to those in the permeability of an individual mesenteric microvessel induced by ATP in a study by $\mathrm{He}$ and Curry, ${ }^{51}$ which also demonstrated the same pattern in endothelial $\mathrm{Ca}^{2+}$ concentration changes. This similarity suggests the mechanism of calcium transient by the FUS-driven MB activities in our study. Further investigation needs to be done to confirm this mechanism.

In summary, in order to find the optimal FUS and MBs that can induce reversible increase in the BBB permeability for the purpose of brain drug delivery, we employed minimally invasive multiphoton microscopy to quantify the BBB permeability to dextran- $155 \mathrm{kDa}$ with similar molecular weight to an antibody. We applyed different doses of FUS in the presence of MBs with an "optimal" size and 
concentration. After several trials, we found the optimal dose of FUS that can transiently increase the BBB permeability. Our analysis was the first study at the individual microvessel level which provides an accurate in vivo assessment for the transient BBB permeability change under the treatment of FUS/MBs. The optimal FUS dose found for the reversible $\mathrm{BBB}$ permeability increase without $\mathrm{BBB}$ disruption is reliable and can be applied to future clinical trials in radioimmunotherapy. Our method can also be used to assess the optimal parameters of FUS/MBs to deliver other types of therapeutic agents and drug carriers.

\section{Acknowledgments}

We thank Dr Adrian Rodriguez-Contreras for his assistance in using the multiphoton imaging system, Dr HoauYan Wang for the oxygen bubbling setup, and Dr Marom Bikson for the function generator. This work was partially supported by NIH-CA153325 and CUNY SEED grant 93348-14-01. RT and JB would like to thank the National Science Foundation (NSF) award NSF/CBET-0967365 for funding.

\section{Author contributions}

All authors contributed toward data analysis, drafting, and revising the paper and agree to be accountable for all aspects of the work.

\section{Disclosure}

The authors report no conflicts of interest in this work.

\section{References}

1. Kramer K, Humm JL, Souweidane MM, et al. Phase I study of targeted radioimmunotherapy for leptomeningeal cancers using intra-Ommaya 131-I-3F8. J Clin Oncol. 2007;25(34):5465-5470.

2. Pardridge WM. Blood-brain barrier drug targeting: the future of brain drug development. Mol Interv. 2003;3(2):90-105, 151.

3. Hawkins BT, Davis TP. The blood-brain barrier/neurovascular unit in health and disease. Pharmacol Rev. 2005;57(2):173-185.

4. Vykhodtseva NI, Hynynen K, Damianou C. Histologic effects of high intensity pulsed ultrasound exposure with subharmonic emission in rabbit brain in vivo. Ultrasound Med Biol. 1995;21(7):969-979.

5. Hynynen K, McDannold N, Vykhodtseva N, Jolesz FA. Noninvasive MR imaging-guided focal opening of the blood-brain barrier in rabbits. Radiology. 2001;220(3):640-646.

6. Yang FY, Wang HE, Lin GL, Lin HH, Wong TT. Evaluation of the increase in permeability of the blood-brain barrier during tumor progression after pulsed focused ultrasound. Int J Nanomedicine. 2012; 7:723-730.

7. Yang FY, Liu SH, Ho FM, Chang CH. Effect of ultrasound contrast agent dose on the duration of focused-ultrasound-induced blood-brain barrier disruption. J Acoust Soc Am. 2009;126(6):3344-3349.

8. Hynynen K, McDannold N, Sheikov NA, Jolesz FA, Vykhodtseva N. Local and reversible blood-brain barrier disruption by noninvasive focused ultrasound at frequencies suitable for trans-skull sonications. Neuroimage. 2005;24(1):12-20.
9. Tran BC, Seo J, Hall TL, Fowlkes JB, Cain CA. Microbubble-enhanced cavitation for noninvasive ultrasound surgery. IEEE Trans Ultrason Ferroelectr Freq Control. 2003;50(10):1296-1304.

10. Konofagou EE. Optimization of the ultrasound-induced blood-brain barrier opening. Theranostics. 2012;2(12):1223-1237.

11. Deng CX. Targeted drug delivery across the blood-brain barrier using ultrasound technique. Ther Deliv. 2010;1(6):819-848.

12. Vlachos F, Tung YS, Konofagou E. Permeability dependence study of the focused ultrasound-induced blood-brain barrier opening at distinct pressures and microbubble diameters using DCE-MRI. Magn Reson Med. 2011;66(3):821-830.

13. Arvanitis CD, Livingstone MS, Vykhodtseva N, McDannold N. Controlled ultrasound-induced blood-brain barrier disruption using passive acoustic emissions monitoring. PLoS One. 2012;7(9):e45783.

14. Liao AH, Liu HL, Su CH, et al. Paramagnetic perfluorocarbon-filled albumin-(Gd-DTPA) microbubbles for the induction of focusedultrasound-induced blood-brain barrier opening and concurrent MR and ultrasound imaging. Phys Med Biol. 2012;57(9):2787-2802.

15. Samiotaki G, Vlachos F, Tung YS, Konofagou EE. A quantitative pressure and microbubble-size dependence study of focused ultrasoundinduced blood-brain barrier opening reversibility in vivo using MRI. Magn Reson Med. 2012;67(3):769-777.

16. Aryal M, Vykhodtseva N, Zhang YZ, Park J, McDannold N. Multiple treatments with liposomal doxorubicin and ultrasound-induced disruption of blood-tumor and blood-brain barriers improve outcomes in a rat glioma model. J Control Release. 2013;169(1-2):103-111.

17. Chen CC, Sheeran PS, Wu SY, Olumolade OO, Dayton PA, KonofagouEE. Targeted drug delivery with focused ultrasound-induced blood-brain barrier opening using acoustically-activated nanodroplets. J Control Release. 2013;172(3):795-804.

18. Chu PC, Chai WY, Hsieh HY, et al. Pharmacodynamic analysis of magnetic resonance imaging-monitored focused ultrasound-induced blood-brain barrier opening for drug delivery to brain tumors. Biomed Res Int. 2013;2013:627496.

19. Samiotaki G, Konofagou EE. Dependence of the reversibility of focused- ultrasound-induced blood-brain barrier opening on pressure and pulse length in vivo. IEEE Trans Ultrason Ferroelectr Freq Control. 2013;60(11):2257-2265.

20. Wang S, Samiotaki G, Olumolade O, Feshitan JA, Konofagou EE. Microbubble type and distribution dependence of focused ultrasound-induced blood-brain barrier opening. Ultrasound Med Biol. 2014;40(1):130-137.

21. Yang FY, Lin YS, Kang KH, Chao TK. Reversible blood-brain barrier disruption by repeated transcranial focused ultrasound allows enhanced extravasation. J Control Release. 2011;150(1):111-116.

22. Park J, Fan Z, Kumon RE, El-Sayed ME, Deng CX. Modulation of intracellular $\mathrm{Ca}^{2+}$ concentration in brain microvascular endothelial cells in vitro by acoustic cavitation. Ultrasound Med Biol. 2010;36(7): $1176-1187$.

23. Shi L, Zeng M, Sun Y, Fu BM. Quantification of blood-brain barrier solute permeability and brain transport by multiphoton microscopy. J Biomech Eng. 2014;136(3):031005.

24. Yuan W, Lv Y, Zeng M, Fu BM. Non-invasive measurement of solute permeability in cerebral microvessels of the rat. Microvasc Res. 2009; 77(2):166-173.

25. Nhan T, Burgess A, Cho EE, Stefanovic B, Lilge L, Hynynen K. Drug delivery to the brain by focused ultrasound induced blood-brain barrier disruption: quantitative evaluation of enhanced permeability of cerebral vasculature using two-photon microscopy. J Control Release. 2013; 172(1):274-280

26. Choi JJ, Feshitan JA, Baseri B, et al. Microbubble-size dependence of focused ultrasound-induced blood-brain barrier opening in mice in vivo. IEEE Trans Biomed Eng. 2010;57(1):145-154.

27. Tung YS, Vlachos F, Feshitan JA, Borden MA, Konofagou EE. The mechanism of interaction between focused ultrasound and microbubbles in blood-brain barrier opening in mice. $J$ Acoust Soc Am. 2011;130(5):3059-3067. 
28. Choi JJ, Wang SG, Tung YS, Morrison B, Konofagou EE. Molecules of various pharmacologically-relevant sizes can cross the ultrasoundinduced blood-brain barrier opening in vivo. Ultrasound Med Biol. 2010;36(1):58-67.

29. Kim SH, Franses EI. New protocols for preparing dipalmitoylphosphatidylcholine dispersions and controlling surface tension and competitive adsorption with albumin at the air/aqueous interface. Colloids Surf B Biointerfaces. 2005;43(3-4):256-266.

30. Feshitan JA, Chen CC, Kwan JJ, Borden MA. Microbubble size isolation by differential centrifugation. J Colloid Interface Sci. 2009;329(2): 316-324.

31. Chen CC, Borden MA. Ligand conjugation to bimodal poly(ethylene glycol) brush layers on microbubbles. Langmuir. 2010;26(16): 13183-13194.

32. Sirsi S, Feshitan J, Kwan J, Homma S, Borden M. Effect of microbubble size on fundamental mode high frequency ultrasound imaging in mice. Ultrasound Med Biol. 2010;36(6):935-948.

33. Sirsi SR, Hernandez SL, Zielinski L, et al. Polyplex-microbubble hybrids for ultrasound-guided plasmid DNA delivery to solid tumors. $J$ Control Release. 2012;157(2):224-234.

34. Garg S, Thomas AA, Borden MA. The effect of lipid monolayer in-plane rigidity on in vivo microbubble circulation persistence. Biomaterials. 2013;34(28):6862-6870.

35. Easton AS, Sarker MH, Fraser PA. Two components of blood-brain barrier disruption in the rat. J Physiol. 1997;503(Pt 3):613-623.

36. Brown RC, Egleton RD, Davis TP. Mannitol opening of the blood-brain barrier: regional variation in the permeability of sucrose, but not $86 \mathrm{Rb}+$ or albumin. Brain Res. 2004;1014(1-2):221-227.

37. Fu BM, Shen S. Acute VEGF effect on solute permeability of mammalian microvessels in vivo. Microvasc Res. 2004;68(1):51-62.

38. Garcia-Villalon AL, Roda JM, Alvarez F, Gomez B, Dieguez G. Carotid blood flow in anesthetized rats: effects of carotid ligation and anastomosis. Microsurgery. 1992;13(5):258-261.

39. Fu BM, Adamson RH, Curry FE. Test of a two-pathway model for small-solute exchange across the capillary wall. Am J Physiol. 1998; 274(6 Pt 2):H2062-H2073.

40. Fu BM, Shen S. Structural mechanisms of acute VEGF effect on microvessel permeability. Am J Physiol Heart Circ Physiol. 2003; 284(6):H2124-H2135.
41. Kimura M, Dietrich HH, Huxley VH, Reichner DR, Dacey RG Jr. Measurement of hydraulic conductivity in isolated arterioles of rat brain cortex. Am J Physiol. 1993;264(6 Pt 2):H1788-H1797.

42. Dreher MR, Liu W, Michelich CR, Dewhirst MW, Yuan F, Chilkoti A. Tumor vascular permeability, accumulation, and penetration of macromolecular drug carriers. J Natl Cancer Inst. 2006;98(5):335-344.

43. Baseri B, Choi JJ, Tung YS, Konofagou EE. Multi-modality safety assessment of blood-brain barrier opening using focused ultrasound and definity microbubbles: a short-term study. Ultrasound Med Biol. 2010;36(9):1445-1459.

44. Mesiwala AH, Farrell L, Wenzel HJ, et al. High-intensity focused ultrasound selectively disrupts the blood-brain barrier in vivo. Ultrasound Med Biol. 2002;28(3):389-400.

45. Kinoshita M, McDannold N, Jolesz FA, Hynynen K. Targeted delivery of antibodies through the blood-brain barrier by MRI-guided focused ultrasound. Biochem Biophys Res Commun. 2006;340(4):1085-1090.

46. McDannold N, Vykhodtseva N, Hynynen K. Targeted disruption of the blood-brain barrier with focused ultrasound: association with cavitation activity. Phys Med Biol. 2006;51(4):793-807.

47. Xia CY, Zhang Z, Xue YX, Wang P, Liu YH. Mechanisms of the increase in the permeability of the blood-tumor barrier obtained by combining low-frequency ultrasound irradiation with small-dose bradykinin. J Neurooncol. 2009;94(1):41-50.

48. Abbott NJ. Role of intracellular calcium in regulation of brain endothelial permeability. In: Pardridge WM, editor. Introduction to the BloodBrain Barrier: methodology, biology and pathology. NY: Cambridge University Press; 1998:345-351.

49. Brown RC, Davis TP. Calcium modulation of adherens and tight junction function: a potential mechanism for blood-brain barrier disruption after stroke. Stroke. 2002;33(6):1706-1711.

50. Paemeleire K, de Hemptinne A, Leybaert L. Chemically, mechanically, and hyperosmolarity-induced calcium responses of rat cortical capillary endothelial cells in culture. Exp Brain Res. 1999;126(4):473-481.

51. He P, Curry FE. Differential actions of cAMP on endothelial $\left[\mathrm{Ca}^{2+}\right] \mathrm{i}$ and permeability in microvessels exposed to ATP. Am J Physiol Heart Circ Physiol. 1993;265(3):H1019-H1023.
International Journal of Nanomedicine

\section{Publish your work in this journal}

The International Journal of Nanomedicine is an international, peerreviewed journal focusing on the application of nanotechnology in diagnostics, therapeutics, and drug delivery systems throughout the biomedical field. This journal is indexed on PubMed Central, MedLine, CAS, SciSearch ${ }^{\circledR}$, Current Contents ${ }^{\circledR} /$ Clinical Medicine,

\section{Dovepress}

Journal Citation Reports/Science Edition, EMBase, Scopus and the Elsevier Bibliographic databases. The manuscript management system is completely online and includes a very quick and fair peer-review system, which is all easy to use. Visit http://www.dovepress.com/ testimonials.php to read real quotes from published authors. 